intensive quit-smoking support as treatment for their disease. The aim of this study was to assess the efficacy of standard quit-smoking interventions (NICE, 2008) for COPD-smokers, to determine levels of support required to improve quit rates.

Methods Current smokers with confirmed COPD were referred from within an inner-city general hospital (inpatients/outpatients) to a dedicated quit-smoking specialist (OSS) or from the community COPD-multidisciplinary team to an integrated OSS, who undertook domiciliary visits for housebound smokers. Both OSS had additional counselling skills. Demographics, disease severity $\left(\mathrm{FEV}_{1}\right)$, smoking history, duration of quit-smoking treatment, pharmacotherapy and quits ( 4 week) were prospectively recorded over 11 months (September 2010-July 2011).

Results 106 patients with moderate COPD M:F 39:67, mean \pm SD age $66.4 \pm 10.4$ y range $\left.49-85 ; \mathrm{FEV}_{1} 1.2 \pm 0.61, \mathrm{n}=76\right)$ were referred: $63(69 \%)$ hospital patients (HP), 43 (41\%) from the community (CP). Compared to the HP who had mean \pm SD FEV $11.4 \pm 0.5 \mathrm{l}$, and smoked $23.5 \pm 11.4$ cigarettes/day on referral, CP had significantly $(\mathrm{p}=0.03)$ worse lung function $\left(\mathrm{FEV}_{1} 1.2 \pm 0.5 \mathrm{l}\right)$ but smoked fewer $(p=0.002)$ cigarettes/day (9.8 $\pm 8 /$ day). $25 / 106(24 \%)$ patients quit, but quits were significantly lower $(p<0.05)$ in the CP $(20 \%)$ compared to the HP (30\%). 45/106 (42\%) were not able to set a quitdate, $5 / 106(5 \%)$ set multiple quit-dates. 56/106 (53\%) used nicotine replacement therapy (NRT), $>2$ products in $48 / 56.18 / 106(17 \%)$ used varenicline, seven sequentially following NRT. Duration of pharmacotherapy for quitters was $6.1 \pm 4.5$ months (mean $\pm \mathrm{SD}$, range $1-16)$. 38/106 (36\%) were discharged after lost to follow-up. Conclusions These data demonstrate that 1 -in- 4 smokers with COPD are able to quit using evidence based tobacco addiction treatment. However, quit rates for these smokers are much lower than the Department of Health $(\mathrm{DH})$ expectation of $>35 \%$, despite intensive interventions by skilled OSS, domiciliary visits to housebound patients, and pharmacotherapy extended beyond the standard 8-12 weeks. Novel approaches, including addressing psychosocial issues, motivational quit-date setting, review of the DH 4-week quit-target and sufficient funding for extended NRT/ Varenicline prescribing, may be required to achieve effective smoking cessation in this patient group.

\section{P126 DOES A PERSONALISED AND NON-CLINICAL EXPLANATION OF LUNG HEALTH TRIGGER THE IMPULSE IN SMOKERS TO MAKE A QUIT ATTEMPT?}

doi:10.1136/thoraxjnl-2011-201054c.126

\section{J L Roberts, I Houghton. NHS Blackpool, Blackpool, UK}

Introduction and Aim A Cochrane review concluded that there is a lack of evidence to support lung function and lung age measures as a method for increasing smoking cessation quit rates. This study aims to assess whether providing lung health checks in workplaces and community settings, combined with immediate access to high quality smoking cessation advice, will promote behaviour change in smokers.

Method The intervention consisted of spirometry followed by a detailed and personalised explanation of the findings. The results were delivered in plain non-clinical language, using lung age, visual tools and local analogies. The results were augmented by a written report and advice with regard to any action indicated. To capitalise on the tension created by the intervention, current smokers were strongly encouraged to seize the moment and have an immediate discussion with a stop smoking adviser who was positioned within easy reach. The smoking status of every individual tested was recorded, along with their age, gender, test results and action advised.
Results 1054 smokers have undertaken the lung health check. 953 were given brief advice to stop smoking. 467 (49\%) subsequently registered with the stop smoking service.

Conclusions Regardless of the spirometry result, whether normal or abnormal, a clear understanding of your lung health appeared to be a powerful motivational trigger and teachable moment for behaviour change. This innovative model potentially provides all the ingredients in one location for promoting smoking cessation as described by Robert West in the 3 Ts strategy: Tension, Trigger, Treatment. ${ }^{1}$ The offer of a lung health check was exceptionally popular in all settings, even among traditionally hard-to-reach groups, and could be targeted according to local need. Joint working with the smoking cessation service improved the outcome for smokers as it took advantage of the immediate situation, triggering the impulse to make a quit attempt. These results justify further work collecting follow-up data to establish whether the trigger of a lung health check converts to a successful long-term quit.

\section{REFERENCE}

1. West R. "Catastrophic" pathways to smoking cessation: findings from national survey. BMJ. 2006; 332:458-60

\section{P127 IMPROVING SMOKING CESSATION ADVICE THROUGH THE IMPLEMENTATION OF A QUALITY IMPROVEMENT INTERVENTION}

doi:10.1136/thoraxjnl-2011-201054c.127

L Hodgson, A Fairhurst, P Thorburn, A F Frew, S R Doffman. Brighton \& Sussex University Hospitals NHS Trust, Brighton, UK

Background Smoking remains the main cause of preventable morbidity and premature death in England ( $\mathrm{DH}$ data) and is estimated to cost the NHS $£ 1.5$ billion a year. Smoking counselling beginning during hospitalisation and including support after discharge increases smoking cessation rates (Rigotti et al 2008). ${ }^{1}$ Health professionals in the hospital are expected to offer cessation advice. An audit was carried out in the acute medical unit of 118 consecutive medical patients which demonstrated that only $1 / 25$ current smokers received any cessation advice. In July 2010, driven by a quality improvement project carried out locally in patients admitted with community-acquired pneumonia, key indicators of high quality care were established, one of which was to clearly document and offer smoking cessation advice to current or recentlyquit smokers. Despite being a requirement, documentation regarding smoking cessation advice was poor. In the respiratory wards, only seven patients were referred to existing smoking cessation services over 6 months. Several interventions were planned to increase awareness. No additional resource was required and members of the multidisciplinary team were employed in a variety of roles. An educational programme was established, including presentations to key specialities (acute and respiratory medicine) and key ward nursing staff. An in-reach programme was developed by the smoking cessation lead nurse, targeting wards where high rates of smoking were identified. Several foundation trainees were employed as "smoking champions," raising awareness among their peers.

Results Since initiation of the interventions, documented cessation advice has steadily risen from $0 \%$ to $68 \%$ of patients with a smoking history (see Abstract P127 figure 1). On the respiratory wards, 77 patients over 6 months (cf seven prior to intervention) were referred to the service. Four-week cessation rates in the patients referred to the cessation service was $82 \%$ and of these patients $70 \%$ had still ceased to smoke at 6 months. 